\title{
ESTIMATION OF COMBINING ABILITY FOR YIELD AND SOME (Zea mays, L.) AGRONOMICAL TRAITS IN NEW YELLOW MAIZE INBRED LINES USING TOP CROSSES METHOD
}

\author{
A.M. Abushosha(1) and M.A.F. Habouh(2) \\ (1) Maize Research Section, Field Crops Research Institute, ARC, Egypt. \\ (2) Department of Agronomy, Faculty of Agriculture, Natural Resources, Aswan University, \\ Aswan 81528, Egypt \\ Received: Feb. 10 , 2019 \\ Accepted: Feb. 14, 2019
}

\begin{abstract}
Fifteen yellow inbred lines of maize were crossed with three yellow inbred testers Gz664, Gm 6061 and Gm 6042 to produce 45 hybrids during summer, 2015 at Gemmeiza Research Station. In summer season, 2016 the 45 hybrids and the three checks were evaluated under two locations i.e Gemmeiza and Nubaria Research Stations. The rustles of these studies can be summered that: mean squares of crosses and their partitions (lines, testers and lines $x$ testers) showed highly significant for all traits under this study except, crosses, line, tester and line $x$ tester mean squares for ear length and ear diameter under two locations. The additive and additive $x$ additive gene actions played more important in the inheritance of ear length at Nub. Location and ear diameter at both locations, Non- additive gene effects played an important role in the inheritance of days to $50 \%$ silking, plant and ear heights and grain yield at both locations and ear length at Gemmeiza location. Regarding $\mathrm{Gm} 17$ had negative and significant GCA effect in Gemmeiza location for plant and ear heights and positively significant for grain yield in both locations, For Gm 19which had positively significant GCA effect for grain yield in both locations $\mathrm{Gm} 37$ had negatively significant GCA effect for days to $50 \%$ silking in both locations and positive and significant GCA effect for grain yield in both locations. For grain yield, the three crosses (Gm17 x Gm 6061), (Gm22 x Gm 6042) and (Gm36 x Gm 6042) had positively highly significant SCA effects in both locations .
\end{abstract}

Key words: (Zea mays L.), Top crosses, combining ability, Genotype x environment, Yellow Maize

\section{INTRODUCTION}

The success of hybrid maize development depends on the ability of the breeding program to rapidly isolate lines that combine well in hybrid combinations and to identify appropriate heterotic combinations to maximize the vigor of the hybrid (Kim and Ajala, 1996). The general process to develop maize hybrids starts with the creation of a source segregating breeding population that is used to develop inbred lines through inbreeding and selection (Betran et al., 2004). Selected inbred lines are then evaluated in hybrid combinations across locations to select superior hybrids and to estimate their combining ability. The behavior of a line in hybrid combination is assessed through the estimation of general combining ability (gca) and specific combining ability (sca) effects. Combining ability analysis is an important method to evaluate the prepotency of cultures to be used in breeding programm and to assess the gene action involved in various characters so as to design an appropriate and efficient breeding method. Combining ability analysis provides these informatios and is frequently used 
by plant breeders to choose parents with a high general combining ability and hybrids with high specific combining ability effects. Numerous investigators reported that the additive gene effects played an effective role in the inheritance of grain yield (Paul and Debanth, 1999; Irshad-El-Haq et al., 2010 and El-Badawy 2013) and number of rows/ear (Mosa et al., 2009; Mosa, 2010 and Aly et al., 2011). While, Kamara (2012), Aly (2013), ElBadawy (2013) and EL-Hosary and Elgammaal (2013) that reported the nonadditive gene effects represented the major role in the inheritance of grain yield and the other agronomical traits. Line $X$ Tester analysis is an extension of the Top cross method in which several testers are used (Kempthorne,1957).

The main aims of this study (a) to estimate of combining ability of inbred lines for grain yield and the other agronomical traits. (b) to identify of type of gen action played an effective role in the inheritance of traits studied and (c) to identify the superior crosses to improve yielding ability in maize breeding program.

\section{MATERIAL AND METHODS}

Fifteen yellow inbred lines of maize; (Gm 10, 12, 14, 15, 17, 19, 20, 22, 23, 25, $35,36,37,41$ and 55 ) were crossed with three yellow inbred testers $\mathrm{Gz} 664$, Gm 6061 and $G m 6042$ to produce 45 hybrids during summer, 2015 at Gemmeiza Research Station name and origin for lines and testers are presented in (Table 1).

The $F_{1}$ of the 45 hybrids and the two standard check hybrids SC 162 and SC 168 were evaluated in randomized block design with four replications during summer, 2016 in the two Experimental Farms at Gemmeiza and Nubaria Research Station. Each entry comprised of one row having 25 plants per row in each replication in a plot size of $4.8 \mathrm{~m}^{2}$. The data ware recorded on six quantitative characters j.e., days to $\mathbf{5 0} \%$ silking plant height, ear height, ear length, ear diameter and grain yield ard. I fed., Line $x$ Tester analysis was carried out using the procedure described by Kempthorne (1957). The superiority percentage was estimated over the two checks as per standard procedure.

Table (1): Name and origin of fifteen yellow inbred lines and three yellow inbred testers.

\begin{tabular}{|l|l|}
\hline \multicolumn{1}{|c|}{ Lines } & \multicolumn{1}{c|}{ Origin } \\
\hline \hline Gm 10, 12, 14, 15, 17, 19, 20, 22, 23 and 25 & (Nub. yellow Pop.) \\
\hline Gm 35, 36, 37, 41 and 55 & (Comp.\#21) \\
\hline Testers & Organ \\
\hline Gz 664 & SD.62MS(M017CW.153) \\
\hline Gm 6061 & Pool-18-627 M \\
\hline Gm 6042 & ( Gm. yellow Pop.) \\
\hline
\end{tabular}




\section{RESULTS AND DISCUSSION}

1- Analysis of variance:

Mean squares for the six traits i.e., (days to $50 \%$ silking, plant height, ear height, ear length, ear diameter and grain yield) under two locations were computed (Table 2) Mean squares of crosses and their partitions (lines, testers and lines $x$ testers) showed highly significant for all traits under this study, except crosses, line, tester and line $x$ tester mean squares for ear length and ear diameter under two locations However, significant mean squares of parents and crosses where its magnitudes were larger than their corresponding mean squares of error would indicate the successful of planned crosses due to the presence of sufficient variability. The contribution of lines $x$ testers interaction which were found to be highly significant, indicating that testers express similar orders of ranking according to the performance of their crosses with the three testers. These results were obtained by Sadek et al. (2001), Gamea et al. (2015) and Aboyousef et al. (2016).

Table (2): Analysis of variances for Days to $50 \%$, Plant height, Ear height, Ear length , Ear diameter and Grain yield traits over two locations.

\begin{tabular}{|c|c|c|c|c|c|c|c|}
\hline \multirow{2}{*}{ S.O.V } & \multirow{2}{*}{ Df } & \multicolumn{2}{|c|}{ Days to $50 \%$ silking } & \multicolumn{2}{|c|}{ Plant height(cm) } & \multicolumn{2}{|c|}{ Ear height(cm) } \\
\hline & & Gm & Nub & Gm & Nub & Gm & Nub \\
\hline Rep & 3 & 0.39 & 2.85 & 240.93 & 718.89 & 84.21 & 233.10 \\
\hline Crosses & 44 & $19.81^{\star \star}$ & $21.66^{\star *}$ & $563.68 * *$ & $425.11^{* *}$ & $542.18^{* *}$ & 443.61* \\
\hline Lines & 14 & $26.08^{\star \star}$ & $19.16^{\star *}$ & $482.88^{\star \star}$ & $370.00 * *$ & $675.73^{\star *}$ & $576.33^{\star *}$ \\
\hline Testers & 2 & $100.51^{\star *}$ & $173.04^{\star *}$ & $675.14^{\star *}$ & $1072.92^{* *}$ & $967.22^{* *}$ & $1902.6^{* *}$ \\
\hline Li $\times T$ & 28 & $10.90^{\star \star}$ & $12.09^{* *}$ & $596.12^{\star *}$ & $406.40^{* *}$ & $445.05^{\star *}$ & $273.03^{* *}$ \\
\hline Error & 308 & 1.09 & 0.93 & 39.51 & 209.99 & 36.49 & 133.01 \\
\hline C.V\% & & 1.88 & 1.56 & 2.80 & 6.84 & 5.37 & 10.48 \\
\hline
\end{tabular}

Table (2): Cont.

\begin{tabular}{|c|c|c|c|c|c|c|c||}
\hline \multirow{2}{*}{ S.O.V } & \multirow{2}{*}{ df } & \multicolumn{2}{|c|}{ Ear length (cm) } & \multicolumn{2}{c|}{ Ear diameter (cm) } & \multicolumn{2}{|c|}{ Grain yield (ard/fad.) } \\
\cline { 3 - 8 } & & Gm & Nub & Gm & Nub & Gm & Nub \\
\hline \hline Rep & 3 & 0.18 & 6.33 & 0.15 & 0.94 & 0.27 & 39.22 \\
\hline Cross & 44 & 2.50 & 1.32 & 0.15 & 0.21 & $77.10^{\star *}$ & $68.41^{\star *}$ \\
\hline Line & 14 & 1.27 & 1.35 & 0.12 & 0.28 & $76.43^{\star *}$ & $66.35^{\star *}$ \\
\hline Tester & 2 & 1.57 & 0.34 & 0.04 & 0.09 & $70.12^{\star *}$ & $100.62^{\star *}$ \\
\hline LI X T & 28 & 3.17 & 1.37 & 0.18 & 0.18 & $77.93^{\star *}$ & $67.14^{\star *}$ \\
\hline \hline Error & 308 & 2.60 & 1.85 & 0.12 & 0.22 & 3.03 & 7.63 \\
\hline CV\% & & 8.31 & 7.27 & 7.16 & 10.83 & 5.76 & 9.14 \\
\hline \hline
\end{tabular}




\section{2- Mean performance}

Regarding Number of days to $50 \%$ silking, all crosses at Gemmeiza location and 41 crosses at Nub. location showed significant difference for earliness compared with SC.162 and 38 crosses at Nub. location showed significant for earliness compared with SC.168.

For plant height mean performance, it showed that out of 45 crosses, 33 crosses had significant and highly significant compared with SC162, 30 crosses had significant and highly significant compared with SC168 in Gemmeiza location, 43 crosses had significant and highly significant compared with check varieties SC162 and 168 at Nub. location.

For ear height mean performance, it showed that out of 45 crosses, 43 crosses had significant and highly significant compared with check varieties SC162 and SC168 at Gemmeiza location, 30 crosses had significant and highly significant compared with SC162 and 19 crosses had significant and highly significant compared with SC168 at Nub. location.

Insignificant differences for all crosses compared with check varieties SC162 and SC168.were optioned for ear length and ear diameter.

Grain yield, at Gemmeiza location three crosses; (Gm $17 \times$ Gm 6061), (Gm $22 \times \mathrm{Gm}$ 6042) and (Gm $37 \times \mathrm{Gz} 664)$ had significant and positive values for grain yield compared with the best check SC168, while at Nub. Location two crosses (Gm $15 \times \mathrm{Gz} 664)$ and (Gm $20 \times$ Gz 664) exhibited significant compered the same check for this trait in (Table 3).

\section{Gene action}

Estimation of genetical parameters for the traits studied under combined data are presented in Table (4). Results cleared that, $\delta^{2}$ GCA-L was more than $\delta^{2}$ GSA-T for ear height at Gemmeiza location for ear length at Nub. Location and grain yield at Gemmeiza location, meaning that most of additive gene action due to lines. These results are in harmony with those reported by Aly et al. (2011), Mousa and Aly (2012) and Aly (2013). The additive and additive $x$ additive gene actions played more locations, while, the non- additive gene effects played an important role in the inheritance of days to $50 \%$ silking, plant and ear heights and grain yield at both locations and ear length at Gemmeiza location. These results agreed with those reported by Todkar and Navale (2006), Singh and Roy (2007), Abdallah et al. (2009), Ibrahim et al. (2010) and Aboyousef et al. (2016). The results in Table (4) revealed that the GSAISCA ratios were less than unity for most studied traits, indicating that SCA variances were more important than GCA variances and that the non-additive variance i.e. dominance, dominance $x$ dominance and additive $x$ dominance gene ainteractions were predominant in the genetic variance of the present genetic materials' for most studied traits, this may illustrate the high values of heterotic effects would be found in these materials besides the divergence of the two parental groups in origin .these results agreed with those reported by Todkar and Navale (2006), Singh and Roy (2007) Abdallah et al. (2009), Ibrahim et al. (2010) and Aboyousef et al. (2016) On the other hand, Aly et al. (2011), Mousa and Aly (2012) and Aly (2013) found that the additive and additive $x$ additive gene actions played an important role in same traits. 


\begin{tabular}{|c|c|c|c|c|c|c|c|c|c|c|c|c|c|c|c|c|c|c|c|c|c|c|c|c|}
\hline 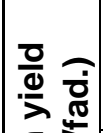 & $\frac{0}{3}$ & 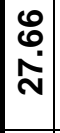 & 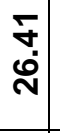 & $\begin{array}{l}\mathscr{2} \\
\stackrel{\leftrightarrow}{\text { N }}\end{array}$ & $\begin{array}{l}\text { L } \\
\text { Oें }\end{array}$ & $\begin{array}{l}0 \\
\text { นุ? } \\
\text { Nิ }\end{array}$ & 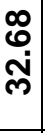 & 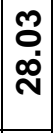 & $\mid \begin{array}{c}\text { N̦ } \\
\stackrel{m}{m}\end{array}$ & 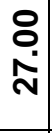 & 竎 & $\begin{array}{l}\text { J } \\
\text { స్ల }\end{array}$ & $\begin{array}{l}\text { Nิ } \\
\text { กิ่ }\end{array}$ & $\begin{array}{l}\text { L̊ } \\
\text { స్ల }\end{array}$ & $\begin{array}{l}7 \\
\infty \\
\infty \\
m\end{array}$ & 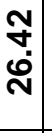 & $\begin{array}{l}\stackrel{9}{a} \\
\dot{m}\end{array}$ & 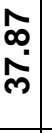 & $\begin{array}{c}\text { フุ } \\
\text { ลิ }\end{array}$ & | & 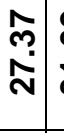 & $\begin{array}{c}8 \\
0 \\
-\dot{m}\end{array}$ & $\begin{array}{l}\stackrel{m}{?} \\
\stackrel{N}{N}\end{array}$ & $\begin{array}{l}\stackrel{\infty}{7} \\
\stackrel{-1}{m}\end{array}$ \\
\hline "ত⿱一兀) & غं. & $\mid \begin{array}{c}m \\
\stackrel{1}{a} \\
\stackrel{2}{N}\end{array}$ & $\mid$ & $\begin{array}{c}0 \\
\text { m. } \\
\text { N }\end{array}$ & $\begin{array}{l}\mathbb{N} \\
\text { N }\end{array}$ & $\begin{array}{l}R \\
\dot{m} \\
\text { mे }\end{array}$ & 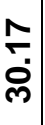 & $\begin{array}{l}1 \\
0 \\
\mathbf{D} \\
\mathbf{N}\end{array}$ & 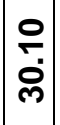 & 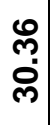 & $\begin{array}{l}\mathfrak{m} \\
\mathfrak{N} \\
\mathfrak{N}\end{array}$ & $\begin{array}{l}8 \\
\emptyset \\
\dot{m} \\
m\end{array}$ & $\mid \begin{array}{c}\tilde{m} \\
\tilde{N} \\
\tilde{N}\end{array}$ & $\begin{array}{c}\stackrel{m}{N} \\
\infty \\
\stackrel{N}{N}\end{array}$ & $\mid \begin{array}{c}\dot{J} \\
\dot{J} \\
\dot{J}\end{array}$ & $\begin{array}{l}0 \\
\stackrel{0}{N} \\
\text { N }\end{array}$ & $\begin{array}{l}\stackrel{0}{0} \\
\ddot{m} \\
\text { | }\end{array}$ & $\begin{array}{l}\tilde{\sigma} \\
\dot{-} \\
\end{array}$ & $\begin{array}{l}\stackrel{0}{ } \\
\text { - } \\
\text { - }\end{array}$ & 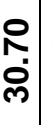 & $\begin{array}{c}\text { ก } \\
\text { Ñ } \\
\text { m. }\end{array}$ & $\begin{array}{l}0 \\
+ \\
\dot{n} \\
\dot{m}\end{array}$ & 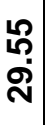 & $\begin{array}{l}\stackrel{m}{\rho} \\
\text { fं }\end{array}$ \\
\hline 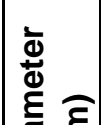 & $\frac{0}{3}$ & $\left|\begin{array}{l|}10 \\
0 \\
\dot{\forall}\end{array}\right|$ & 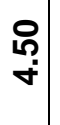 & $\begin{array}{l}\text { டி } \\
+ \\
\dot{f}\end{array}$ & $\stackrel{\text { ஸ̊ }}{+}$ & $\begin{array}{l}\stackrel{q}{q} \\
\dot{f}\end{array}$ & 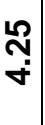 & 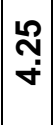 & $\underset{\stackrel{P}{+}}{\dot{f}}$ & $\begin{array}{l}\stackrel{\rho}{m} \\
\dot{v}\end{array}$ & $\stackrel{\text { L̊ }}{\stackrel{\text { mे }}{\dot{\gamma}}}$ & 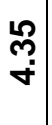 & 官 & $\stackrel{\text { L̊ }}{+}$ & 年 & 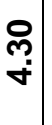 & $\begin{array}{l}\stackrel{P}{+} \\
\dot{f}\end{array}$ & $\begin{array}{l}\stackrel{o}{+} \\
\dot{\nabla}\end{array}$ & $\begin{array}{l}\stackrel{m}{\text { f }} \\
\dot{f}\end{array}$ & 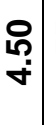 & \begin{tabular}{l|l}
$\stackrel{2}{\circ}$ \\
$\stackrel{1}{*}$
\end{tabular} & 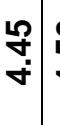 & $\begin{array}{l}\stackrel{0}{\circ} \\
\stackrel{7}{+}\end{array}$ & $\stackrel{+}{+}$ \\
\hline జٓ & छे| & $\mid \begin{array}{l}\qquad \\
0 \\
\dot{\gamma} \\
\dot{\gamma}\end{array}$ & $\begin{array}{l}\stackrel{R}{1} \\
\dot{f}\end{array}$ & 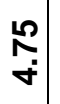 & $\begin{array}{l}\stackrel{P}{1} \\
\dot{f}\end{array}$ & $\begin{array}{l}\stackrel{L}{R} \\
\dot{\tau}\end{array} \mid$ & $\begin{array}{l}\stackrel{P}{P} \\
\dot{f}\end{array}$ & $\begin{array}{l}0 \\
\infty \\
\dot{f}\end{array}$ & \begin{tabular}{|l|}
$\mathbf{L}$ \\
0 \\
$\dot{\gamma}$ \\
\end{tabular} & $\begin{array}{l}\text { 오 } \\
\dot{\gamma}\end{array}$ & \begin{tabular}{|l|}
$\stackrel{2}{0}$ \\
$\dot{q}$
\end{tabular} & $\begin{array}{l}\stackrel{L}{\infty} \\
\stackrel{+}{\dot{f}}\end{array}$ & शి & 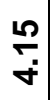 & 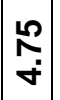 & 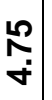 & $\begin{array}{l}\text { P } \\
\dot{f}\end{array}$ & $\begin{array}{l}\stackrel{1}{2} \\
+ \\
\dot{\sim}\end{array}$ & $\begin{array}{l}\stackrel{8}{\infty} \\
\dot{q}\end{array}$ & $\begin{array}{l}\qquad \\
0 \\
\dot{\sim}\end{array}$ & $\begin{array}{c}\stackrel{1}{\infty} \\
\dot{\sim} \\
\dot{*}\end{array}$ & $\begin{array}{l}f \\
\dot{f}\end{array}$ & $\begin{array}{l}\stackrel{\llcorner}{\infty} \\
\infty \\
\dot{f}\end{array}$ & 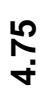 \\
\hline 点 & $\frac{0}{\bar{z}}$ & 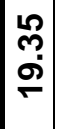 & $\begin{array}{l}R \\
0 \\
\rightarrow \\
\rightarrow\end{array} \mid$ & 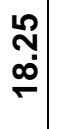 & $\begin{array}{c}\text { ని } \\
\text { ô } \\
\text { | }\end{array}$ & $\begin{array}{l}\frac{1}{2} \\
\infty \\
\rightarrow \\
\rightarrow\end{array}$ & 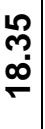 & 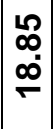 & 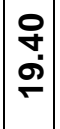 & 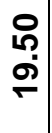 & 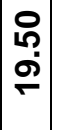 & 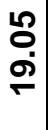 & 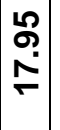 & $\begin{array}{c}0 \\
9 \\
\infty \\
-1\end{array}$ & 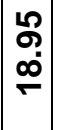 & $\begin{array}{c}R \\
0 \\
\infty \\
G\end{array}$ & $\mid$ & $\begin{array}{l}2 \\
0 \\
0 \\
-1 \\
-1\end{array}$ & $\begin{array}{l}8 \\
\text { هి }\end{array}$ & 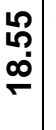 & 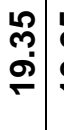 & 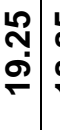 & 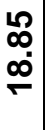 & 국 \\
\hline 焉 & غं & 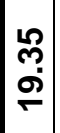 & \begin{tabular}{c}
0 \\
$m$ \\
$\infty$ \\
\hdashline \\
-
\end{tabular} & $\begin{array}{l}q \\
q \\
\sigma \\
\sim\end{array}$ & 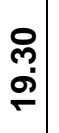 & 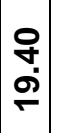 & 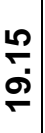 & $\begin{array}{l}0 \\
\tilde{m} \\
\stackrel{-}{\mathrm{g}}\end{array}$ & 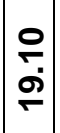 & $\begin{array}{l}8 \\
0 \\
\text { î. }\end{array}$ & 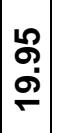 & $\begin{array}{l}\text { Ñ } \\
\text { ○ } \\
\text { N }\end{array}$ & 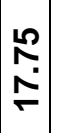 & $\begin{array}{l}\text { L } \\
\text { î. }\end{array}$ & 占 & $\begin{array}{l}\text { స్ } \\
\text { హे }\end{array}$ & $\begin{array}{c}\mathfrak{L} \\
0 \\
\infty \\
-1\end{array}$ & 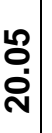 & 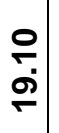 & 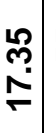 & 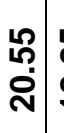 & $\begin{array}{c}2 \\
\infty \\
\infty \\
\infty \\
-1 \\
-1\end{array}$ & $\begin{array}{l}\text { O } \\
\vdots \\
\stackrel{0}{0}\end{array}$ & 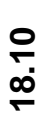 \\
\hline$\frac{\vec{t}}{\sigma}$ & $\frac{0}{3}$ & 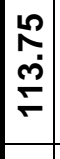 & 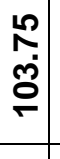 & $\begin{array}{l}8 \\
\text { ه } \\
8\end{array}$ & 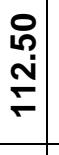 & $\begin{array}{l}0 \\
\text { กิ } \\
\text { స్ }\end{array}$ & 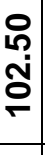 & 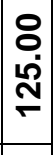 & $\begin{array}{c}\stackrel{1}{\sim} \\
\mathfrak{m} \\
\stackrel{9}{7}\end{array}$ & $\begin{array}{c}\stackrel{1}{\sim} \\
\mathfrak{m} \\
\underset{7}{7}\end{array}$ & 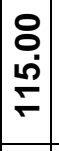 & 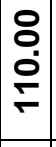 & 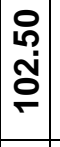 & 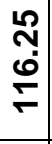 & 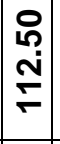 & 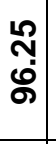 & 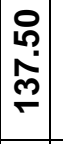 & 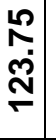 & $\begin{array}{l}\stackrel{1}{N} \\
\infty \\
\mathscr{\infty}\end{array}$ & 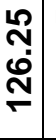 & 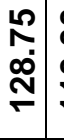 & 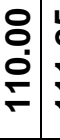 & $\begin{array}{l}\text { Ñ } \\
\underset{7}{-} \\
\end{array}$ & 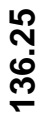 \\
\hline พึ & نं & $\mid \begin{array}{c}\stackrel{2}{\sim} \\
\tilde{m} \\
\stackrel{7}{-1}\end{array}$ & 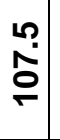 & 足 & 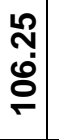 & $\begin{array}{c}10 \\
\mathcal{N} \\
\mathcal{M} \\
\stackrel{-}{\mid}\end{array} \mid$ & 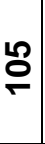 & $\begin{array}{l}\stackrel{2}{\hat{N}} \\
\mathfrak{\sim} \\
\mathfrak{\sim} \\
\end{array}$ & 농 & 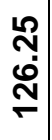 & 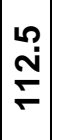 & 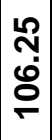 & $\mid \begin{array}{c}\mathbf{L} \\
0 \\
0 \\
0 \\
-\end{array}$ & 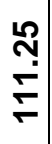 & 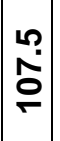 & 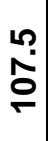 & 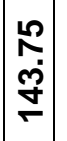 & 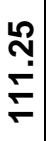 & 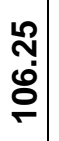 & 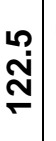 & 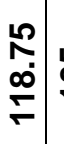 & $\stackrel{2}{\circ}$ & 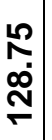 & 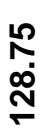 \\
\hline$\frac{\vec{c}}{\bar{d}}$ & $\frac{0}{\bar{z}}$ & 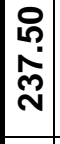 & 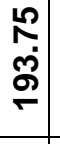 & 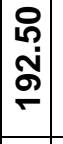 & 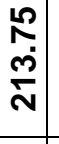 & $\begin{array}{l}\text { N } \\
\text { N } \\
\text { N̦ }\end{array}$ & $\begin{array}{l}\stackrel{2}{N} \\
-\mathfrak{N} \\
\end{array}$ & 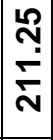 & 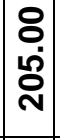 & 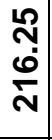 & 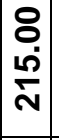 & 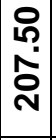 & 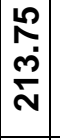 & 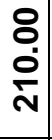 & 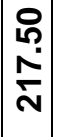 & $\begin{array}{l}\text { గి } \\
\text { N్} \\
\text { సે }\end{array}$ & 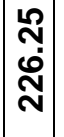 & $\begin{array}{l}\mathfrak{R} \\
\infty \\
\stackrel{\infty}{\mathbf{N}} \\
\text { Nat }\end{array}$ & $\begin{array}{l}\text { N } \\
\text {-̇ } \\
\text { N }\end{array}$ & $\begin{array}{l}\text { గొ } \\
\text { Nิ } \\
\text { Nat }\end{array}$ & $\begin{array}{l}\text { గొ } \\
\text { N్} \\
\text { N̦ }\end{array}$ & 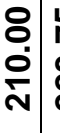 & 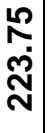 & $\begin{array}{l}\text { in } \\
\text { กิ }\end{array}$ \\
\hline$\frac{\bar{\sigma}}{\alpha}$ & نं & $\begin{array}{l}\text { : } \\
\text { N̦ } \\
\text { N̦}\end{array}$ & $\begin{array}{l}0 \\
\stackrel{\circ}{n} \\
\end{array}$ & 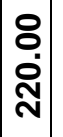 & 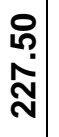 & $\left|\begin{array}{l}0 \\
\stackrel{0}{0} \\
\multirow{2}{*}{} \\
\mid\end{array}\right|$ & $\begin{array}{l}8 \\
\text { ¿̦ } \\
\text { స్ }\end{array}$ & 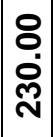 & 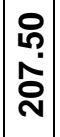 & 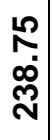 & $\begin{array}{l}\text { \& } \\
\text { N̦} \\
\text { N. }\end{array}$ & 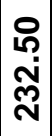 & 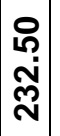 & 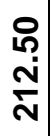 & 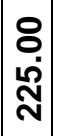 & $\begin{array}{l}n \\
\text { م } \\
\infty \\
D^{\circ}\end{array}$ & 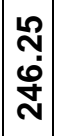 & 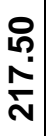 & \begin{tabular}{l} 
م \\
\multirow{N}{N}{} \\
N
\end{tabular} & \begin{tabular}{l}
$\stackrel{2}{N}$ \\
\multirow{N}{N}{} \\
ans
\end{tabular} & 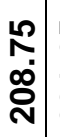 & $\begin{array}{c}\stackrel{2}{N} \\
-1 \\
\end{array}$ & 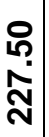 & $\begin{array}{l}\text { N } \\
\text { ஸे } \\
\text { N }\end{array}$ \\
\hline 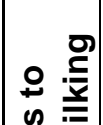 & $\frac{0}{\bar{z}}$ & 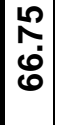 & $\begin{array}{l}8 \\
0 \\
0 ٌ 0 \\
0\end{array}$ & 号 & $\begin{array}{l}8 \\
0 \\
\dot{0} \\
\end{array}$ & $\begin{array}{c}\text { Ln } \\
\text { กี } \\
\text { ర్ }\end{array}$ & $\begin{array}{l}\text { م } \\
\text { ป̂. }\end{array}$ & $\begin{array}{c}n \\
N \\
\mathcal{M} \\
0\end{array}$ & $\left|\begin{array}{l}8 \\
\dot{-1} \\
0\end{array}\right|$ & $\begin{array}{l}8 \\
\dot{0} \\
0\end{array}$ & 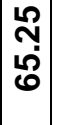 & $\begin{array}{c}8 \\
\dot{-} \\
6\end{array}$ & $\begin{array}{l}8 \\
8 \\
8 \\
10\end{array}$ & $\begin{array}{l}0 \\
0 \\
0 \\
0 \\
0\end{array}$ & $\begin{array}{c}8 \\
\text { ปิ } \\
\text { d }\end{array}$ & 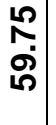 & 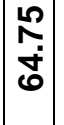 & & $\begin{array}{l}\text { L̊ } \\
\text { ¿े }\end{array}$ & $\begin{array}{l}12 \\
0 \\
0 \\
\end{array}$ & $\begin{array}{l}\text { ధุ } \\
\text { ల్ర }\end{array}$ & $\begin{array}{l}8 \\
\text { : } \\
\text { iी }\end{array}$ & & ¿̊ \\
\hline ๑ จे & نं & $\mid \begin{array}{l}10 \\
\infty \\
\infty \\
10\end{array}$ & $\begin{array}{l}\text { 오 } \\
\text { مึٌ } \\
\text { | }\end{array}$ & $\begin{array}{l}8 \\
\text { กิ } \\
\text { กิ }\end{array}$ & 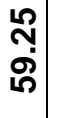 & 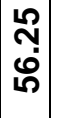 & 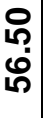 & 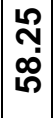 & 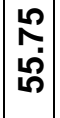 & $\begin{array}{c}\stackrel{L}{N} \\
\\
\text { กิ }\end{array}$ & م્ & 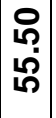 & 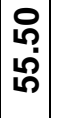 & $\begin{array}{l}\stackrel{1}{1} \\
\infty \\
\infty \\
0\end{array}$ & 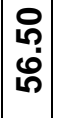 & $\begin{array}{l}\stackrel{2}{2} \\
\text { กิ } \\
\end{array}$ & $\begin{array}{l}8 \\
0 \\
0 \\
i n\end{array}$ & 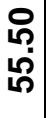 & 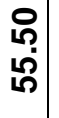 & 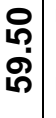 & $\begin{array}{l}\text { ஸึ } \\
\text { ஸी }\end{array}$ & & 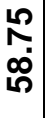 & 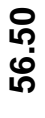 \\
\hline : & $\begin{array}{l}0 \\
0 \\
0 \\
\mathscr{y} \\
0 \\
\\
\end{array}$ & 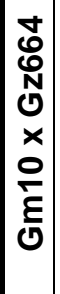 & 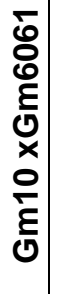 & 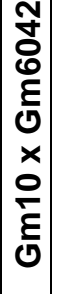 & 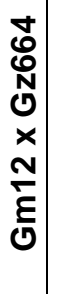 & 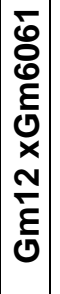 & 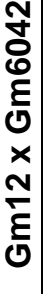 & 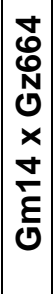 & 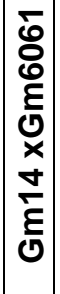 & 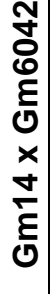 & 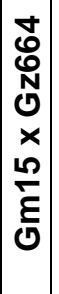 & 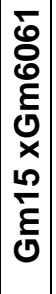 & 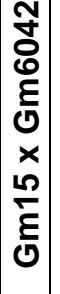 & 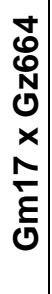 & 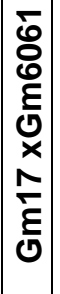 & 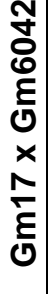 & 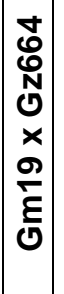 & $\begin{array}{l}-1 \\
0 \\
0 \\
\varepsilon \\
0 \\
x \\
- \\
\vec{\varepsilon} \\
0\end{array}$ & 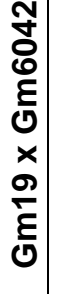 & 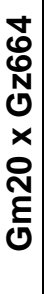 & 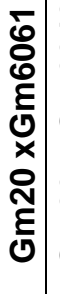 & 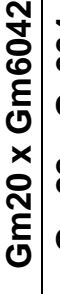 & 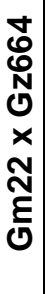 & 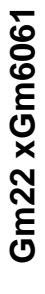 \\
\hline
\end{tabular}




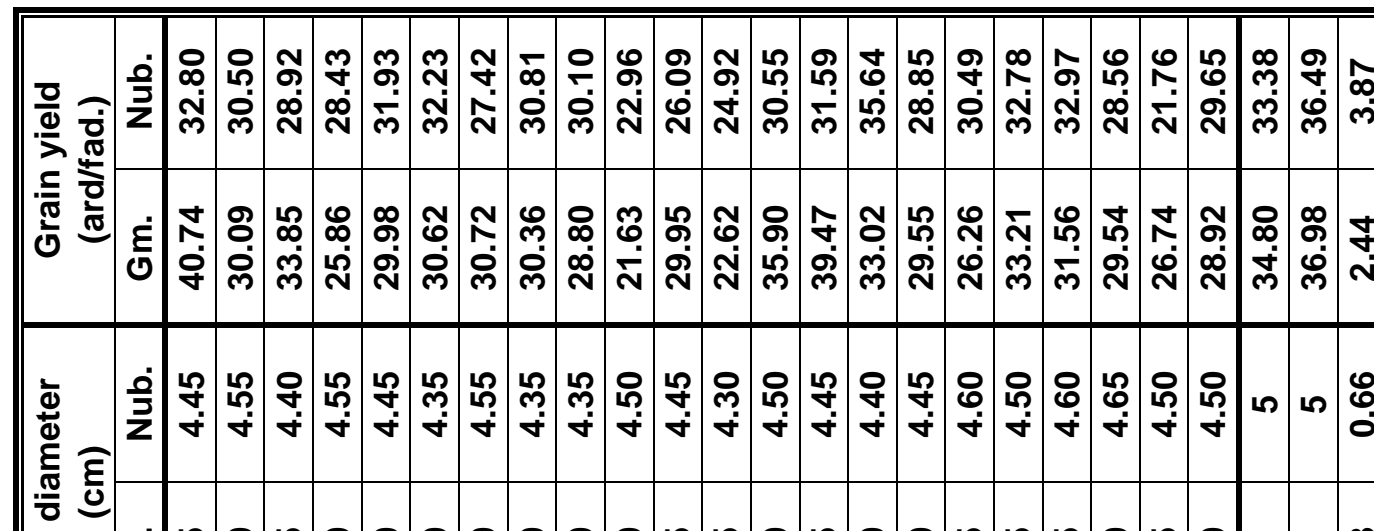

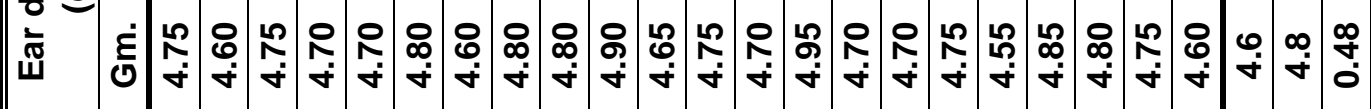

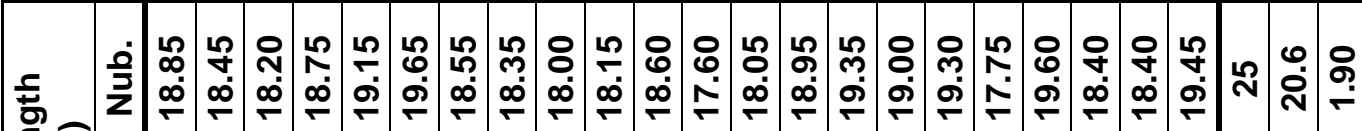

ब

离

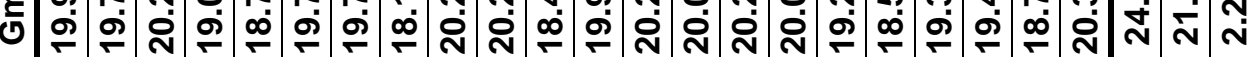

- $\quad$ 논

志 ய்

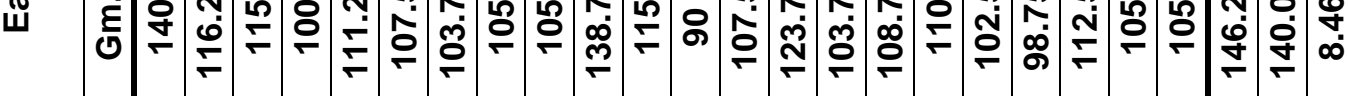

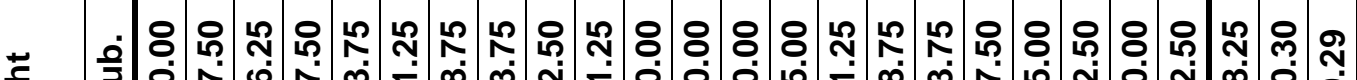

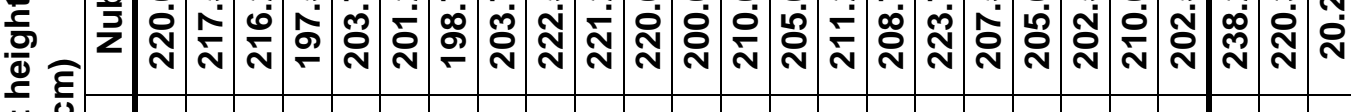
䒕

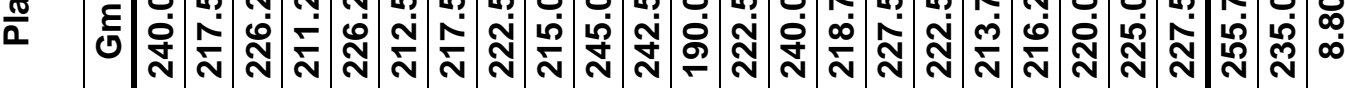

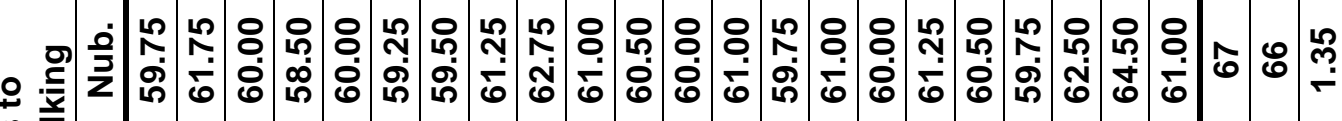

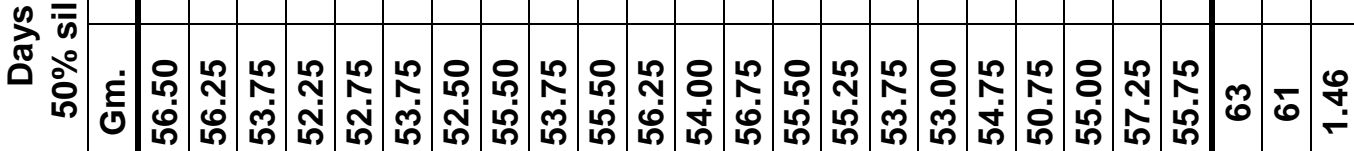

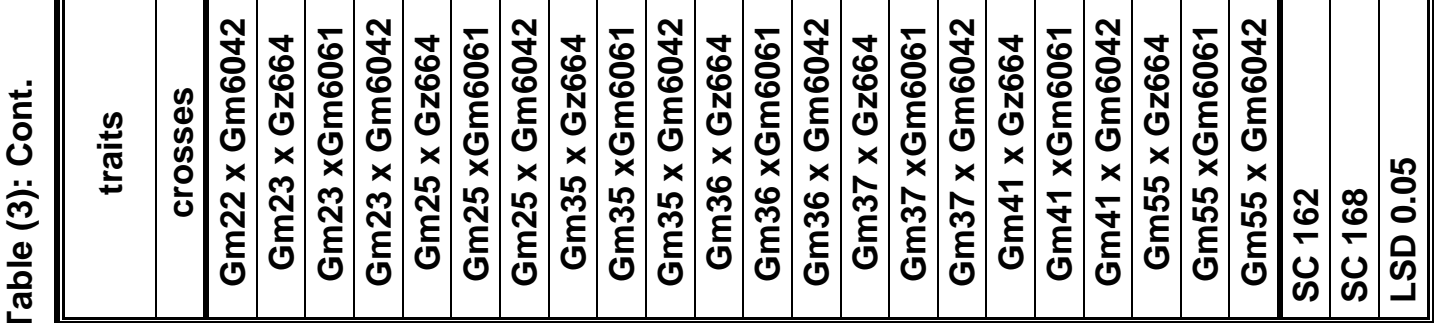


Table (4): Estimates of the variance due to general combining ability (GCA), specific combining ability (SCA) and their interaction with locations for Days to 50\%, Plant height, Ear height, Ear length, Ear diameter and Grain yield .

\begin{tabular}{|c|c|c|c|c|c|c|c|c|c|c|c|c|}
\hline \multirow[t]{2}{*}{ S.0.V } & \multicolumn{2}{|c|}{$\begin{array}{c}\text { Days to } \\
50 \% \text { silking }\end{array}$} & \multicolumn{2}{|c|}{$\begin{array}{l}\text { Plant height } \\
\text { (cm) }\end{array}$} & \multicolumn{2}{|c|}{$\begin{array}{c}\text { Ear height } \\
\text { (cm) }\end{array}$} & \multicolumn{2}{|c|}{$\begin{array}{l}\text { Ear length } \\
\qquad \text { (cm) }\end{array}$} & \multicolumn{2}{|c|}{$\begin{array}{l}\text { Ear diameter } \\
\qquad(\mathrm{cm})\end{array}$} & \multicolumn{2}{|c|}{$\begin{array}{l}\text { Grain yield } \\
\text { (ard/fad.) }\end{array}$} \\
\hline & Gm & Nub & Gm & Nub & Gm & Nub & Gm & Nub & Gm & Nub & Gm & Nub \\
\hline$\sigma^{2} L$ & 1.27 & 0.59 & -9.44 & -3.03 & 19.22 & 25.28 & -0.158 & -0.002 & -0.005 & 0.008 & -0.125 & -0.066 \\
\hline$\sigma^{2} T$ & 1.49 & 2.68 & 1.32 & 11.11 & 8.70 & 27.16 & -0.027 & -0.017 & -0.002 & -0.002 & -0.130 & 0.558 \\
\hline$\sigma^{2} G C A$ & 0.09 & 0.09 & -0.31 & 0.18 & 0.93 & 1.63 & -0.006 & -0.001 & 0.000 & 0.000 & -0.008 & 0.012 \\
\hline$\sigma^{2} s c a$ & 2.45 & 2.79 & 139.15 & 49.10 & 102.14 & 35.01 & 0.143 & -0.120 & 0.015 & -0.010 & 18.725 & 14.878 \\
\hline GCA/SCA & 0.036 & 0,032 & $-0,0022$ & 0,0037 & 0,0091 & 0,046 & $-0,042$ & , 0083 & - & - & $-0,0004$ & 0,0008 \\
\hline
\end{tabular}

All negative estimates of variance were considered zero

\section{General combining ability effects $\left(g^{\wedge}\right)$ :}

High positive values of GCA effects would be of interest in most traits, while for heading date, ear height and plant height, high negative values would be useful from the plant breeder point of view

Estimation of GCA effects $\left(g^{\wedge}\right)$ for the fifteen yellow maize inbred lines and the three testers was tabulated in Table (6). For inbred line, Gm 10 had negative and significant GCA effects in plant height in location Gemmeiza and both locations for ear height. For $\mathrm{Gm} 15$, it had positively significant GCA effects for grain yield in location Nub. Regarding Gm 17, had negative and significant SCA effects effects in location Gemmeiza for plant and ear heights and positively significant SCA effects for grain yield in both locations.

For Gm 19 had positively significant GCA effects for grain yield in both location.

Regarding Gm 20, it had negative and significant GCA effects for plant height and positively significant GCA effects for grain yield at location Gemmeiza.

For Gm 23, it had negatively significant GCA effects for days to $50 \%$ silking in both locations and plant height in Gemmeiza location.

Regarding Gm 25, it had negatively significant GCA effects for days to $50 \%$ silking, plant and ear heights in both locations.

For Gm 35, it had negative and significant GCA effects for days to $50 \%$ silking in location Gemmeiza and ear height in Nub location.

Regarding Gm 36, it had negatively significant GCA effects for days to $50 \%$ silking in Nub., plant and ear heights in Gemmeiza location.

For Gm 37, it had negatively significant GCA effects for days to $50 \%$ silking in both locations and positive and significant for grain yield in both plant locations.

Regarding Gm 41, it had negatively significant GCA effects for days to $50 \%$ silking in both locations and plant and ear heights in Gemmeiza location. 


\section{Testers:}

The results showed that the tester $\mathrm{Gz}$ 664 had highly positive and significant GCA effects for grain yield in Nub location. Gm 6061 had negatively significant GCA effects for plant and ear heights in Gemmeiza location and had positively significant for grain yield in Gemmeiza location and Gm 6042 had negatively significant GCA effects for days to $\mathbf{5 0} \%$, silking, plant height in Nub location. and ear height in location Gemmeiza. (Table 5).

\section{Specific combining ability effects} $\left(\mathbf{s}^{\wedge}{ }_{\mathrm{ij}}\right)$ :

Specific combining ability effect of the 45 top crosses for traits studied are presented in Table (7) appeared that, number of days to $50 \%$ silking, five crosses at both locations,(Gemmeiza and Nub.); ( Gm10 x Gm 6042), ( Gm17 x Gm 6042), (Gm20 x Gm 6042), ( Gm25 x Gz 664 ) and ( Gm55 x Gz 664), had desirable negative and significant values of $\left(s^{\wedge}{ }_{i j}\right)$, While, six crosses at Gemmeiza location; (Gm14 x Gm 6061), ( Gm15 x Gm 6061), (Gm19 x Gm 6061), ( Gm35 x Gm 6061), (Gm36 x Gm 6061) and ( Gm41 x Gz 664) and four crosses at Nub. Location; (Gm12 x Gm 6061), ( Gm15 x Gm 6042),
(Gm35 x Gz 664) and ( Gm37 x Gz 664) showed regarding to negative significant for $\left(s^{\wedge}{ }_{i j}\right)$ towards earliness.

For plant height 14 crosses; ( $G \mathrm{~m} 12 \mathrm{x}$ Gz 664), ( Gm12 x Gm 6042), (Gm14x Gm 6061), ( Gm15 x Gz 664), ( Gm17 x Gm 6042), ( Gm19 x Gm 6061) (Gm19 x Gm 6042), ( Gm20 x Gm 6061), ( Gm22 x Gz 664), ( $\mathrm{Gm} 23 \times \mathrm{Gm}$ 6042), (Gm35 x Gz 664), ( Gm35 x Gm 6061), ( Gm36 x Gm 6061) and ( Gm55 x Gz 664) had negative and significant for $\left(\mathrm{s}^{\wedge}{ }_{\mathrm{ij}}\right)$ towards shortness in Gemmeiza location, while, the cross $(\mathrm{Gm} 10 \times \mathrm{Gm} 6061)$ had negative and significant $\left(s^{\wedge}{ }_{i j}\right)$ towards shortness in Nub. location.

For ear height, tow crosses (Gm19 $x$ $\mathrm{Gm}$ 6042) and (Gm22 x Gz 664) had highly negatively and significant SCA effects $\left(s^{\wedge}{ }_{i j}\right)$ in Gemmeiza and Nub. Locations, while, 10 crosses; (Gm10x Gm 6042), (Gm12 x Gz 664), (Gm12x Gm 6042), (Gm14 x Gm 6061), ( Gm19 x Gm 6061), (Gm20 x Gm 6042), ( Gm23 x Gm 6042), (Gm35 x Gz 664), ( $\mathrm{Gm} 35 \times \mathrm{Gm}$ 6061) and (Gm36 x Gm 6061) had negative and significant values of $\left(s^{\wedge}{ }_{i j}\right)$ for low ear position in Gemmeiza location.

Table (5): General combining ability (GCA) effects of the three inbred lines as testers for grain yield and the other traits studied under two locations.

\begin{tabular}{|c|c|c|c|c|c|c|c|c|c|c|c|c|c|}
\hline \multirow{2}{*}{\multicolumn{2}{|c|}{ Testers }} & \multicolumn{2}{|c|}{$\begin{array}{c}\text { Days to } \\
50 \% \text { silking }\end{array}$} & \multicolumn{2}{|c|}{$\begin{array}{l}\text { Plant height } \\
\text { (cm) }\end{array}$} & \multicolumn{2}{|c|}{$\begin{array}{l}\text { Ear height } \\
\text { (cm) }\end{array}$} & \multicolumn{2}{|c|}{$\begin{array}{l}\text { Ear length } \\
\text { (cm) }\end{array}$} & \multicolumn{2}{|c|}{$\begin{array}{c}\text { Ear } \\
\text { diameter } \\
(\mathbf{c m})\end{array}$} & \multicolumn{2}{|c|}{$\begin{array}{l}\text { Grain yield } \\
\text { (ard/fad.) }\end{array}$} \\
\hline & & Gm. & Nub. & Gm. & Nub. & Gm. & Nub. & Gm. & Nub. & Gm. & Nub. & Gm. & Nub. \\
\hline \multicolumn{2}{|c|}{ Gz664 } & $1.34^{* *}$ & 1.61 & $2.52^{\star *}$ & 3.75 & $4.611^{* *}$ & 3.97 & -0.18 & 0.01 & -0.02 & 0.02 & $-0.783^{* *}$ & $1.111^{* *}$ \\
\hline \multicolumn{2}{|c|}{ Gm6061 } & -0.09 & 0.17 & $-3.81^{\star *}$ & 0.83 & $-2.722^{\star *}$ & 2.47 & 0.14 & -0.08 & 0.03 & -0.04 & $1.233^{* *}$ & 0.311 \\
\hline \multicolumn{2}{|c|}{ Gm6042 } & $-1.24^{\star *}$ & $-1.78 * *$ & 1.29 & $-4.58^{*}$ & $-1.89 *$ & -6.44 & 0.04 & 0.07 & -0.01 & 0.02 & $-0.450^{*}$ & $-1.422^{* \star}$ \\
\hline \multirow[t]{2}{*}{ Lsd gi } & 0.05 & 0.6 & 0.25 & 1.61 & 3.70 & 1.54 & 2.95 & - & - & - & - & 0.44 & 0.71 \\
\hline & 0.01 & 0.78 & 0.32 & 2.09 & 4.81 & 2.00 & 3.83 & - & - & - & - & 0.58 & 0.92 \\
\hline \multirow{2}{*}{$\begin{array}{l}\text { Lsd } \\
\text { gi- gj }\end{array}$} & 0.05 & 0.85 & 0.35 & 2.27 & 5.24 & 2.18 & 4.17 & - & - & - & - & 0.63 & 1.0 \\
\hline & 0.01 & 1.10 & 0.45 & 2.95 & 6.80 & 2.83 & 5.41 & - & - & - & - & 0.82 & 1.3 \\
\hline
\end{tabular}




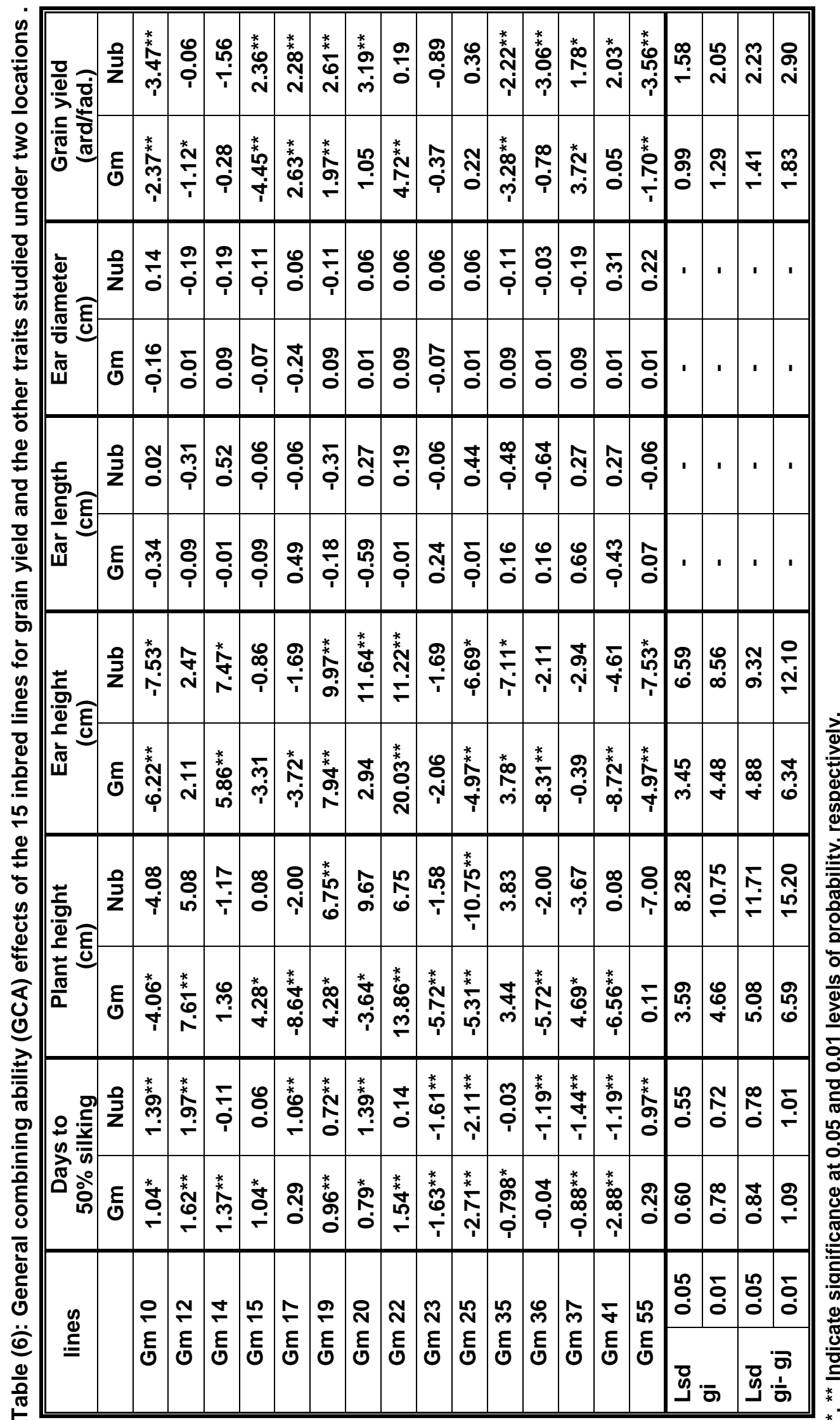




\section{A.M. Abushosha and M.A.F. Habouh}

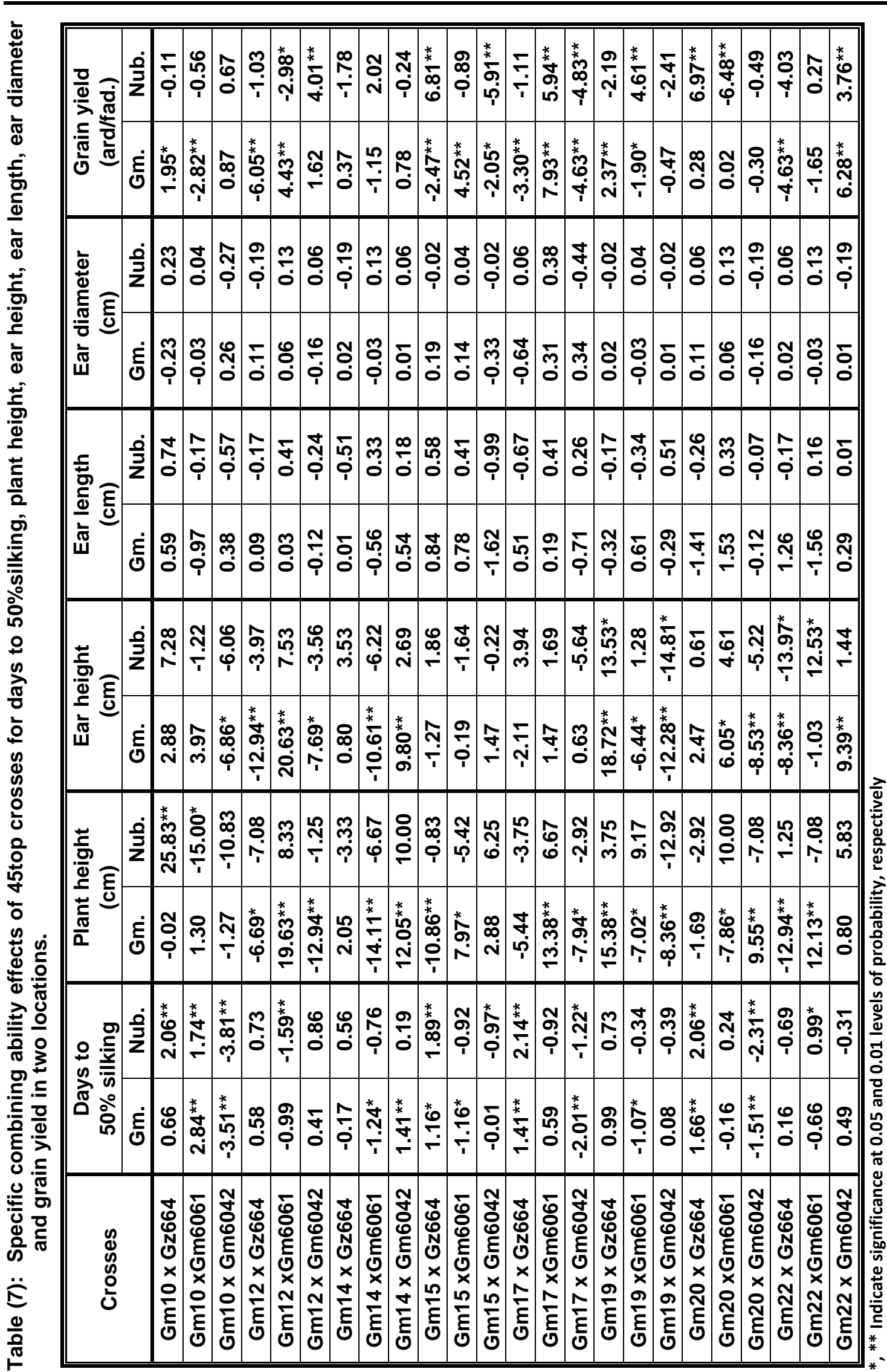




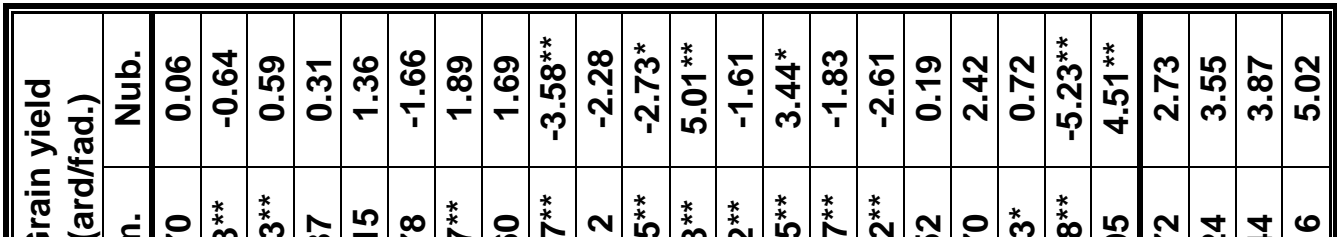

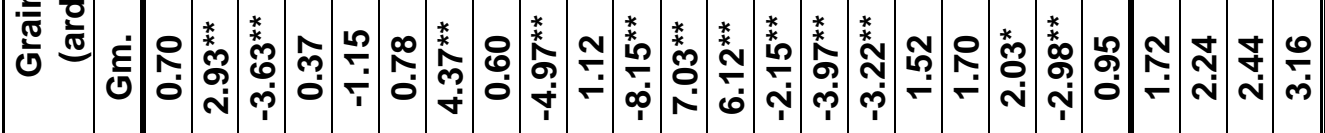

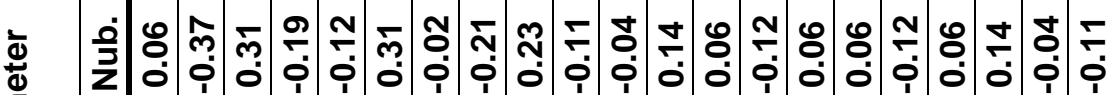

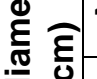

๘

ш U

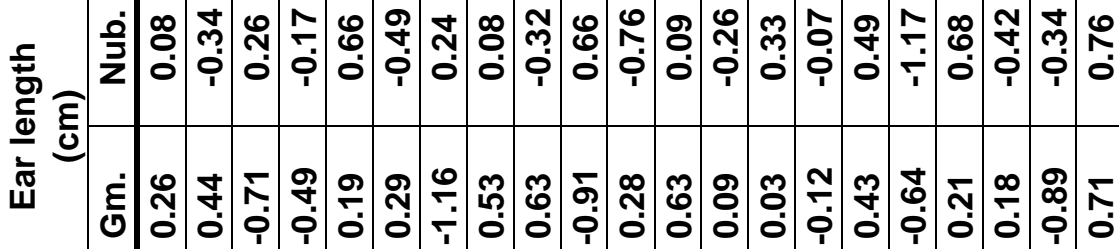

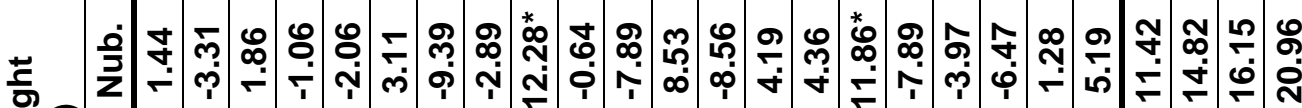

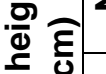

芫

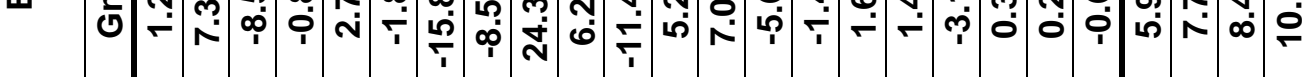

Z $\quad$ ำ

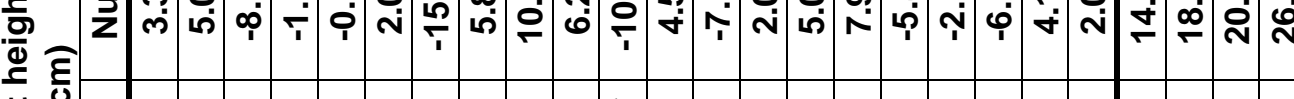
䓠

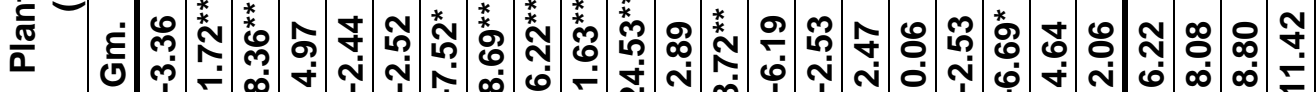

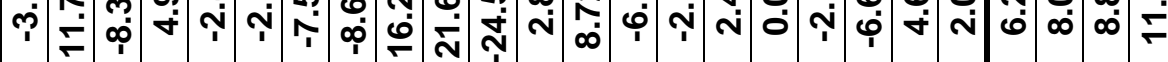

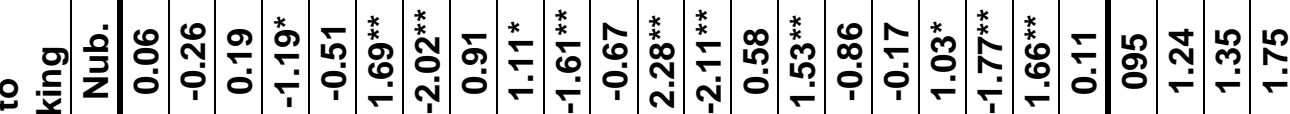

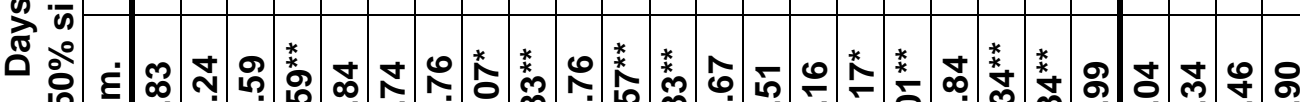
ん)

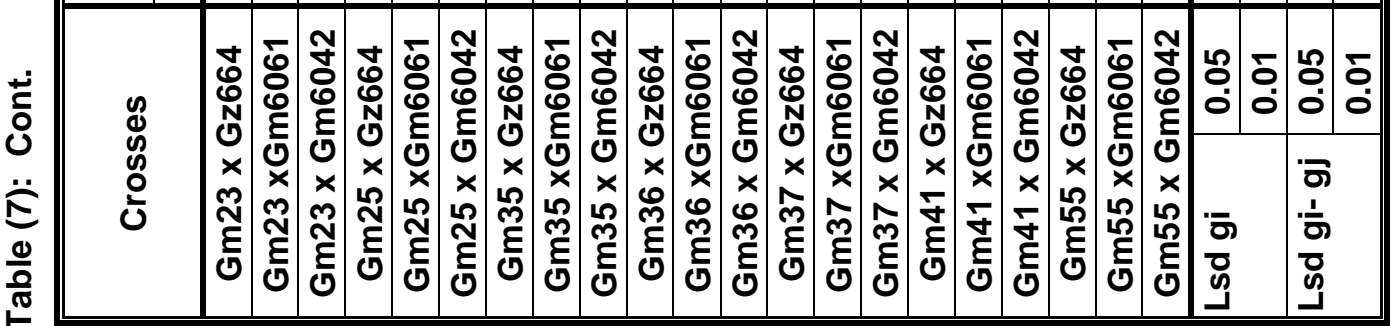


With respect to grain yield, three crosses (Gm17 x Gm 6061) , (Gm22 x Gm 6042) and (Gm36 $x$ Gm 6042) had positively highly significant SCA effects in both locations. Eight crosses; ( $\mathrm{Gm} 10 \mathrm{x}$ Gz 664), (Gm12 x Gm 6061), (Gm15 x Gm 6061), (Gm19 x Gz 664), (Gm23 x Gm 6061), (Gm35 x Gz 664) , (Gm37 x Gz 664) and (Gm55 x Gz 664) exhibited highly positively significant SCA effects at Gemmeiza location, six crosses; ( $G$ m12 $x$ Gm 6042), (Gm15 x Gz 664), (Gm19 x Gm 6061), (Gm20 x Gz 664), (Gm37x Gm 6061) and (Gm55x Gm 6042) these crosses had the best combiners for $\operatorname{SCA}\left(\mathbf{s}^{\wedge}{ }_{i j}\right)$.

The parent showing high SCA in most important traits could be used in hybrid cultivar breeding when anailable, while the other parents exhibiting high GCA; possessing high amounts of additive genetic variance are more suitable for selection programs in the later generations.

Finally, it could be concluded that further improvement in earliness, plant and ear heights (towards shortness) and grain yield are still possible.

\section{REFERENCES}

Abd allah, T.A.E., A.I. Gabr Afaf and A.A. El Kheshen (2009). Combining ability in line $x$ tester crosses of maize (Zea mays L.) Annals of Agric. Sci., Moshtohor, 47 (1): 11 - 20.

Aboyousef, H.A., H.A.A., Gamea and Moshera S.E. Sadek (2016). Evaluation of some new white maize top crosses for yield and some other traits. Alex. J. Agric. Sci. 61, (4) pp. 409 - 418.

Aly RSH (2013). Relationship between combining ability of grain yield and yield components for some newly yellow maize inbred lines via line $x$ tester analysis. Alex. J. Agric. Res. 58(2): 115-124.
Aly, R.S.H., Metwali E.M.R. and SThM Mousa (2011). Combining ability of maize (Zea mays L.) inbred lines for grain yield and some agronomic traits using top cross mating design. Glob. J. Mole. Sci. 6(1): 01-08.

Betran, J., M. Menz and M. Bänziger (2004). Corn breeding. In:"Corn: Origin, History, Technology, and Production" (Eds. Smith, C. W.; Betran, J. and Runge, E. C. A.), John Wiley and Sons, Hoboken, NJ., 305395.

El-Badawy, M.E.M. (2013). Heterosis and combining ability in maize using diallel crosses among seven new inbred Lines. Asian J Crop Sci. 5(1): 113.

EL-Hosary, A.A.A. and A.A. Elgammaal (2013). Utilization of line $x$ tester model for evaluating the combining ability of some new white maize inbred lines. Egypt. J. Plant Breed. 17(1): 79-72.

Gamea, H.A.A., M.A.M. EL-Ghonemy and H.A. Aboyousef (2015). Analysis of combining ability in new yellow maize inbred lines (Zea mays L.). Egypt. J. of Appl. Sci., 30 (1): 65 - 76.

Ibrahim, K.H.A.M., M.A. Abd El-moula and M.E.M. Abd El-Azeem (2010). Combining ability analysis of some yellow maize (Zea mays L.) inbred lines. Egypt. J. Agric. Res., 88 (1) : 29 $-46$.

Irshad-El-Haq, M., Ajmal SU, Munir M,M. Gulfaraz (2010). Gene action studies of different quantitative traits in maize. Pak J Bot. 42: 1021-1030.

Kamara, M.M. (2012). Heterosis, combining ability and molecular studies on maize breeding. PhD Thesis Fac Agric Kafr El-Sheikh Univ Egypt.

Kempthorne, O. (1957). An Introduction to Genetic Statistics. John Wily \& sons Inc., New York. 
Kim, S. K. and S. O. Ajala (1996). Combining ability of tropical maize germplasm in West Africa II. Tropical vs Temperate $\times$ Tropical origins. Maydica, 41:135-141.

Mosa, H.E., A.A. Motawei and A.A. ElShenawy (2009). Selection of new single crosses of maize for grain yield and resistance to downy mildew disease under different locations and potassium fertilization. J Agric Res Kafr El-Sheikh Univ. 35(2): 522-536.

Mosa, H.E. (2010). Estimation of combining ability of maize inbred lines using top cross mating design. J. Agric. Res. Kafr EL-Sheikh univ., 36(1):1-14.

Mousa, S.Th.M. and R.S.H. Aly (2012). Estimation of combining ability effects of new white maizeinbred lines (Zea mays L.) via line $x$ tester analysis.
Fourth Field Crops Conference "Field Crops Facing Future Challenges". Egy. J.Agric. Res., 90(4): 77-90

Paul, K.K. and S.C. Debanth (1999). Combining ability analysis in maize. Pakistan J Sci Ind Res. 42: 141-144.

Sadek, S.E., M.S.M. Soliman and A.A. Barakat (2001). Evaluation of newly developed maize lines using commercial inbred testers. Egypt. J. Appl. Sci.16:406-425.

Singh, P.K. and A.K. Roy (2007). Diallel analysis of inbred lines in maize (Zea mays L.). Intl. J. Agric. Sci. 3(1):213216.

Todkar, L. P. and P. A. Navale (2006). Selection of parents and hybrids through combining ability studies in maize. J. Maharashtra Agric. Univ., India. 31 (3): 264 - 267. 
تقدير القدرة على التالف لمحصول الحبوب وبعض الصفات الزراعية فى سلالات الذرة الثامية الصفراء الجديدة باستخدام طريقة الهجن القمية

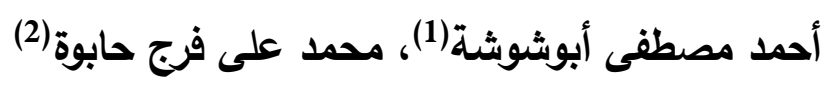

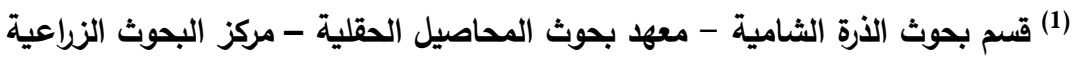

(2) قسم المحاصيل- كلية الززاعة والموارد الطبيعية - جامعة اسوان النيان

الملغص العربي

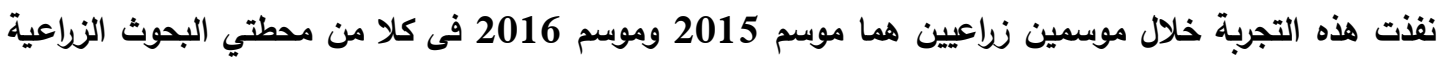

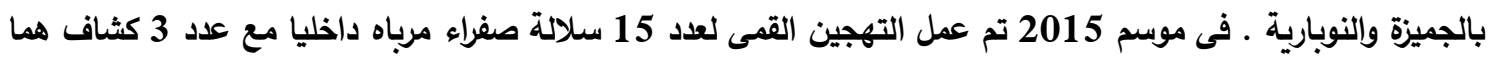

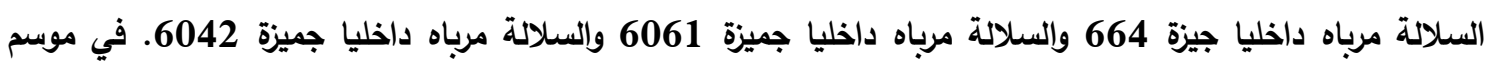

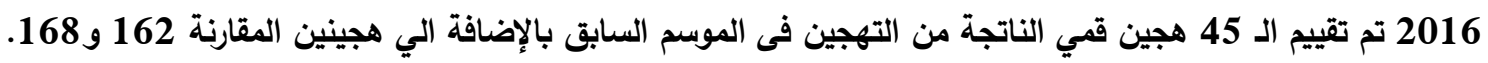

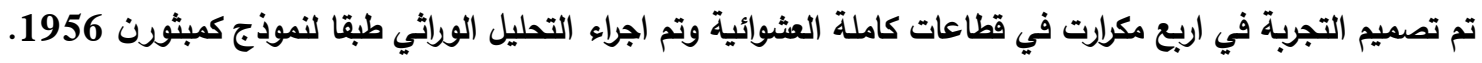

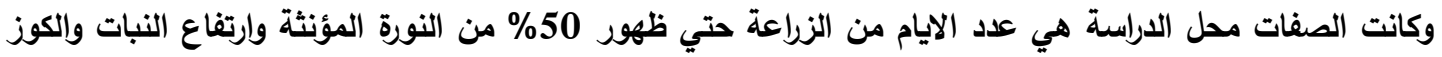

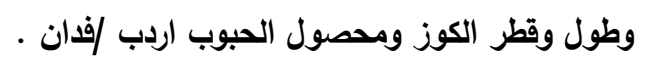

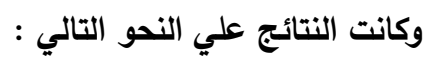

1. 1 تحليل التباين الراجع إلى المواقع عالي المعنوية لجميع الصفات محل الدارسة.

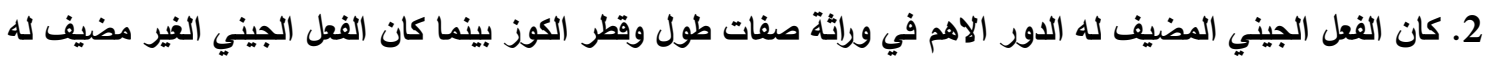

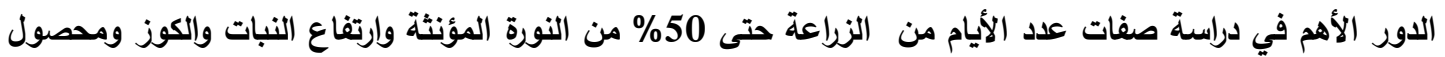
الحبوب اردب للفذان.

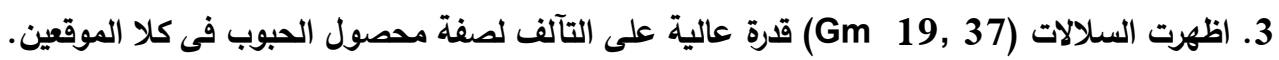

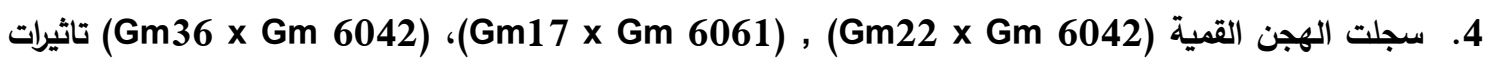
عالية المنوية للقدرة الخاصة على التالف0 0

\footnotetext{
أسماء السبادة المحكمين

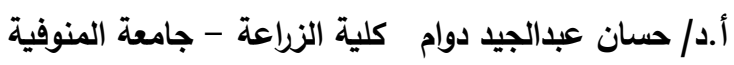

